\title{
Nanoscaled Multilayer Thin Films Based on GZO
}

\author{
Ching-Hsuang Cheng ${ }^{a}$, Wan-Yu Wu ${ }^{b}$, Jyh-Ming Ting ${ }^{c^{*}}$ \\ Department of Materials Science and Engineering \\ National Cheng Kung University, Tainan, Taiwan \\ a fumiya@yahoo.com.tw \\ ${ }^{\mathrm{b}}$ wanyu@mail.mse.ncku.edu.tw \\ $c^{*}$ jting@mail.ncku.edu.tw
}

received paper 16.11.2007, received revised paper 18.12.2007, accepted date, 2.01.2008

Keywords: Gallium; Multilayer; Optical properties; Zinc oxide

\begin{abstract}
Nanoscaled gallium-doped ZnO (GZO) thin films, bi-layer Pt/GZO thin films, and tri-layer $\mathrm{GZO} / \mathrm{Pt} / \mathrm{GZO}$ thin films were prepared and their characteristics were investigated. These films were deposited on glass substrates using either $\mathrm{rf}$ or dc magnetron sputter deposition. The deposition time and the target-to-substrate distance were varied to obtain different total film thicknesses and layer thicknesses. Effects of total film and layer thicknesses on the optical properties and the electrical properties were studied. Theoretical calculations were performed to discuss effect of the thickness on the optical transmittance of the GZO film. As-deposited GZO films show high electrical resistivity, which was greatly reduced by 2 to 3 orders of magnitude due to the introduction of a surface layer of Pt film. However, the optical transmittance was also reduced. This was improved by using an addition anti-refractive GZO surface layer on the Pt/GZO. A GZO/Pt/GZO film exhibiting visible light transmittance greater than $75 \%$ and electrical resistivity in the order of $10^{-4} \mathrm{ohm}-\mathrm{cm}$ was obtained.
\end{abstract}

\section{Introduction}

The development of transparent conductive oxide (TCO) thin films and coatings has been of great interest as they are an important integral part of a number of electro-optical devices. For example, they are used as electrodes to convey electrical signals which are crucial for the operations of the devices. They are also used in other applications such as optical filters, heat mirrors, low emittance films for advanced glazing, protective coating, and decorative coatings. These TCO thin films include Sn-doped indium oxides (ITO) [1,2,3], Al-doped zinc oxides (AZO) [2,4], Ga-doped zinc oxides (GZO)[2,5,6], and F-doped tin oxides (FTO)[7]. As-deposited TCO thin films often exhibit less than desirable electrical resistivity as well as optical transmittance. To improve the electrical and optical properties, TCO thin films are deposited at elevated temperatures or subjected to post-deposition various forms of heat treatments. However, when low melting point substrates are used, high-temperature deposition and heat treatments are not viable. Efforts have therefore been made to improve the electrical conductivity via the development of multilayer structures consisting of TCO/metal $/ \mathrm{TCO}$.

A majority of studies on multilayer structures were to develop electrodes for use in flat panel displays. These multilayer structures include $\mathrm{ZnS} / \mathrm{Ag} / \mathrm{ZnS}$ films[8,9,10], ITO/Ag/ITO films[11,12], $\mathrm{IZO} / \mathrm{AgPd}$ films[13], oxide/Ag alloy/-oxide/Ag alloy/oxide where the oxide is either ITO or Sn-doped $\mathrm{CdO}$ (ICO) films[14], and $\mathrm{ZnO} / \mathrm{Al} / \mathrm{ZnO}$ films[15]. Three-layer $\mathrm{TiO}_{2} / \mathrm{Ag} / \mathrm{TiO}_{2}$, three-layer $\mathrm{ZnS} / \mathrm{Ag} / \mathrm{ZnS}$, and five-layer $\mathrm{TiO}_{2} / \mathrm{Ag} / \mathrm{TiO}_{2} / \mathrm{Ag} / \mathrm{TiO}_{2}$ were also investigated for application in heat mirrors[16,17]. 
Furthermore conductive transparent thin films of $\mathrm{TiO}_{2} / \mathrm{Ag} / \mathrm{TiO}_{2}$ were investigated for use as electrodes in dye sensitizing solar cells[18]. It appears that $\mathrm{Ag}$ is a popular metal used in various multilayer structures. However, a disadvantage is that defects originating from Ag layer corrosion were observed[19,20]. Therefore, based on the consideration of thermal stability, Pt is used in this study as the metallic mid-layer. Also, to have better electrical properties, Ga doped $\mathrm{ZnO}$, i.e., GZO, is used as the TCO layer. In this study, thin films of GZO, Pt/GZO, and GZO/Pt/GZO were prepared and investigated for their optical as well as electrical properties.

\section{Experimental}

GZO thin films were deposited on glass substrates at room temperature using rf magnetron sputter deposition. The GZO target was a 3-inch 5\% Ga doped ZnO. The rf power and working pressure were respectively $150 \mathrm{~W}$ power and $10 \mathrm{mTorr}$. Three working distances or target-substrate distances were used. They are $5 \mathrm{~cm}, 6 \mathrm{~cm}$ and $6.5 \mathrm{~cm}$. Different deposition times $(1 \mathrm{~min}, 3 \mathrm{~min}, 5 \mathrm{~min}, 7 \mathrm{~min}, 10 \mathrm{~min}$, and $15 \mathrm{~min}$ ) were used. Pt thin films were then deposited on the GZO films using dc magnetron sputter deposition to form bi-layer Pt/GZO films. The working pressure and dc power used for the Pt thin film deposition were $10 \mathrm{~m}$ Torr and 20W, respectively. The 3-inch Pt target has a purity of $99.99 \%$. Different deposition times $(30 \mathrm{sec}, 45 \mathrm{sec}, 60 \mathrm{sec}$, and $75 \mathrm{sec}$ ) were used to obtain Pt films with different thicknesses. Finally, GZO thin films having various thicknesses were deposited on the bilayer Pt/GZO films to form tri-layer GZO/Pt/GZO films. The tri-layer GZO/Pt/GZO films therefore have different combinations of layer thickness. The surface morphology of the resulting films was analyzed using high resolution scanning electron microscopy (HRSEM). The crystallinity was examined using glazing incident x-ray diffractometry (GIXD). The film thickness was determined using $\alpha$-step profilometry and spectroscopic ellipsometry. The refractive indices and distinction coefficients of the films were also obtained from spectroscopic ellipsometry measurements. The optical properties of GZO, Pt/GZO, and GZO/Pt/GZO thin films were investigated using UV-Vis optical spectroscopy. Finally, the electrical resistivity was determined using a 4-point probe.

\section{Results and discussion}

All the GZO obtained exhibit a hexagonal structure with a preferred (002) orientation. The grain size and the surface roughness of GZO films increase with the film thickness. The thickness of GZO thin films obtained at different deposition conditions was found to increase linearly with the deposition time. Fig. 1 shows the thickness of GZO thin films obtained at a working distance was $5 \mathrm{~cm}$. The thickness was determined using $\alpha$-step and ellipsometry, both of which give almost identical results. GZO thin films obtained at working distances longer than $5 \mathrm{~cm}$ are thinner due to the thermalization effect[21]. The optical transmittances of GZO thin films obtained at different working distances are also different.

Fig. 2A shows the optical transmittances of GZO films obtained at working distances of 5 $\mathrm{cm}, 6 \mathrm{~cm}$, and $6.5 \mathrm{~cm}$. The thicknesses of

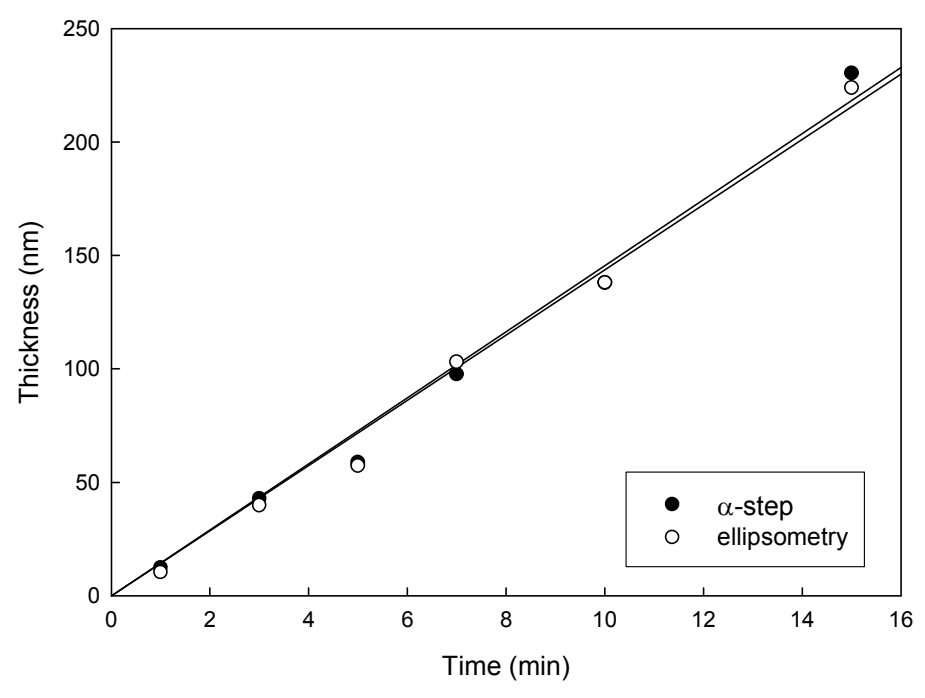

Fig. 1 Thickness as a function of deposition time 
these films are respectively $58 \mathrm{~nm}, 55 \mathrm{~nm}$, and $53 \mathrm{~nm}$. Due to the nanoscale thicknesses, which are less than the skin depths, these films exhibit UV transmittances. The skin depth is approximately $160 \mathrm{~nm}$ for $\mathrm{ZnO}[22]$. The UV transmittance increases with decreasing film thickness. Meanwhile, the UV transmittance decreases with the electromagnetic radiation frequency due to a reduced skin depth at a higher frequency. In the visible range, all the GZO films exhibit optical transmittances that are greater than $80 \%$. It appears that a thinner film has slightly better visible light transmittance seemly due to less absorption. However, such a thickness effect cannot be overlooked by just considering the absorption. Fig. 2B shows the optical transmittances at $500 \mathrm{~nm}$ for GZO thin films having different thicknesses. These films were obtained at $\mathrm{rf}=150 \mathrm{~W}$ power, pressure $=10$ mTorr, working distance $=5 \mathrm{~cm}$, and

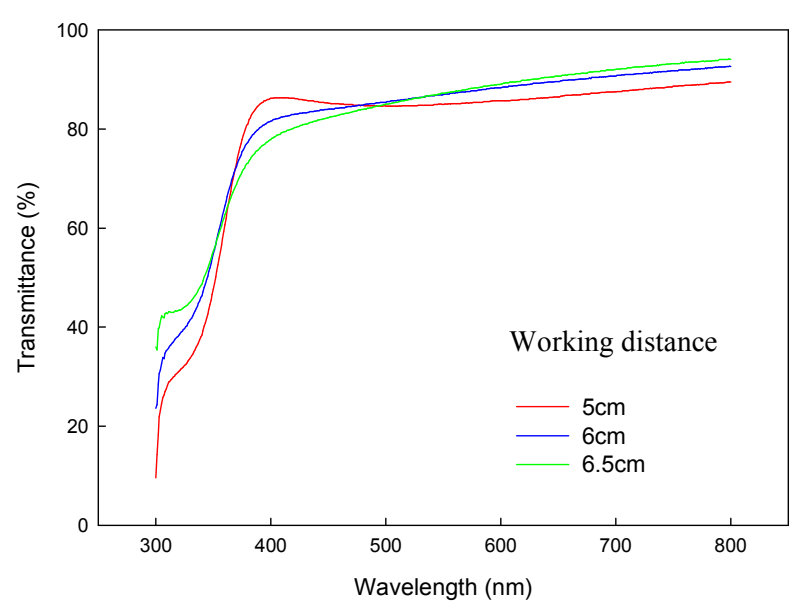

(A)

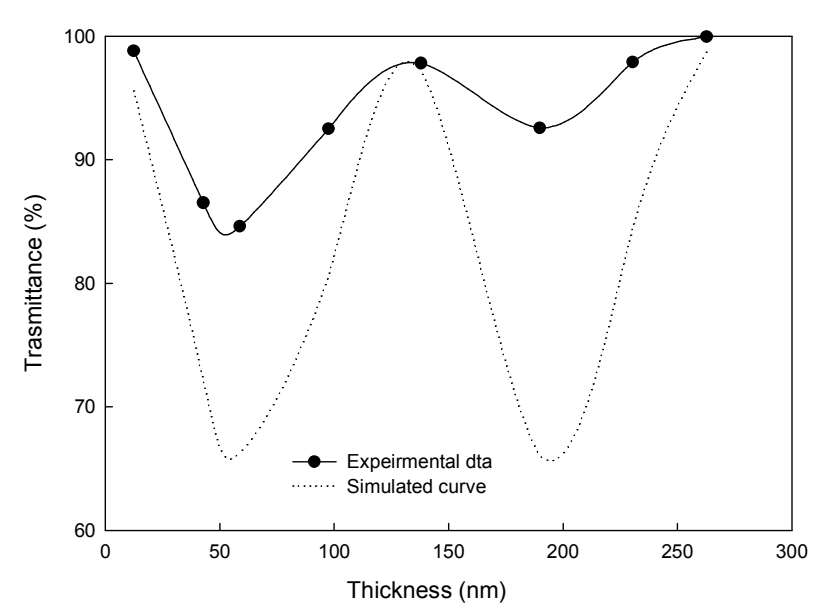

(B)

Fig. 2 Optical transmittances of GZO thin films obtained at different (A) working distances and (B) Deposition times. Only the optical transmittances at $500 \mathrm{~nm}$ are shown in (B).

various deposition times. The filled circles and the solid line represent respectively the experimental data and the best fit. It is seen that the transmittance varies up and down with the thickness.

Optical absorption alone cannot explain the data. The optical transmittance, $\mathrm{T}$, across a single boundary can be described by the following equation ${ }^{12}$

$$
T=\frac{I_{t} \cos \theta_{t}}{I_{i n} \cos \theta_{i n}}=\frac{n_{t} \cos \theta_{t}}{n_{i n} \cos \theta_{i n}}\left(\frac{E_{t}}{E_{i n}}\right)^{2}
$$

where $I_{t}$ is the transmitted flux density, $I_{\text {in }}$ the incident flux density, $\theta_{t}$ the refracted angle, $\theta_{\text {in }}$ the incident angle, $n_{t}$ the refractive index of transmitting medium, $n_{i n}$ the refractive index of incident medium, $E_{t}$ the electric field in the transmitting medium, and $E_{i n}$ the electric field in the incident medium. For more than one boundaries, the relationship between the incident wave and the transmitted wave is

$$
\left[\begin{array}{c}
E_{1} \\
H_{1}
\end{array}\right]=M_{1} M_{2} \cdots M_{n}\left[\begin{array}{c}
E_{n+1} \\
H_{n+1}
\end{array}\right]
$$


$\mathrm{E}_{\mathrm{j}}$ and $\mathrm{H}_{\mathrm{j}}$ are respectively the electric and magnetic fields across boundary $\mathrm{j}(\mathrm{i}=1,2$, to $\mathrm{n}+1)$.

$M_{j}=\left[\begin{array}{cc}\cos k_{0} h & \left(i \sin k_{0} h\right) / \mathrm{Y}_{j} \\ \mathrm{Y}_{j} i \sin k_{0} h & \cos k_{0} h\end{array}\right]$ is called the material characteristic matrix of the medium between boundary $\mathrm{j}$ and $\mathrm{j}+1$. $k_{0} h$ is the phase change across the medium where $k_{0}=2 \pi / \lambda$ ( $\lambda$ the wavelength) and $h=\widetilde{n} d \cos \theta_{j}(\tilde{n}=n+i k$ is the complex form of the refractive index and $\mathrm{d}$ is the thickness of the medium). $Y_{j}=\sqrt{\varepsilon_{0} / \mu_{0}} \cdot\left(\widetilde{n}_{j} \cos \theta_{j}\right)$ and $\sqrt{\varepsilon_{0} / \mu_{0}} \cdot\left(\widetilde{n}_{j} / \cos \theta_{j}\right)$ when the electric field is perpendicular and parallel to the plane-of-incidence, respectively. In $\mathrm{Y}_{\mathrm{j}}, \varepsilon_{0}$ is the electric permittivity of free space and $\mu_{0}$ is the permeability of free space. Therefore, assuming that all the boundaries are perfect, the optical transmittance can be calculated using Eq. (1) where $E_{i n}$ and $E_{t}$ are determined using Eq. (2). The $n / k$ values measured in this study were used in the calculations and $n_{t}=n_{i n}=1$ for the air. Also both $\theta_{\mathrm{i}}$ and $\theta_{\mathrm{t}}$ are equal to 1 in this study. The calculated results are represented by the dotted line shown in Fig. 2B. Although the experimental data do not quite agree with the calculated data, possibly due to the non-isotropicness of GZO and the imperfect boundaries, the wavy trends are clearly similar.

Although the GZO films obtained exhibit optical transmittances greater than $80 \%$, the electrical resistivity ranges from $\sim 10^{-1}$ to $\sim 10^{-2}$ $\mathrm{ohm}-\mathrm{cm}$, which are not quite desirable. However, with a surface layer of nanoscale Pt film, the electrical resistivity is largely reduced, as shown in Fig. 3. The electrical resistivity of the 22-nm GZO film is $0.78 \mathrm{ohm}-\mathrm{cm}$, which is reduced by 2 to 3 orders of magnitude due to the presence of a surface Pt film.

The reduction of the electrical resistivity due to the addition of a $\mathrm{Pt}$ surface layer can be explained by the so-called parallel model[23]. While the surface $\mathrm{Pt}$ film reduces the electrical resistivity, it also reduces the optical transmittance as shown in Fig. 4. The thicker the Pt film is the

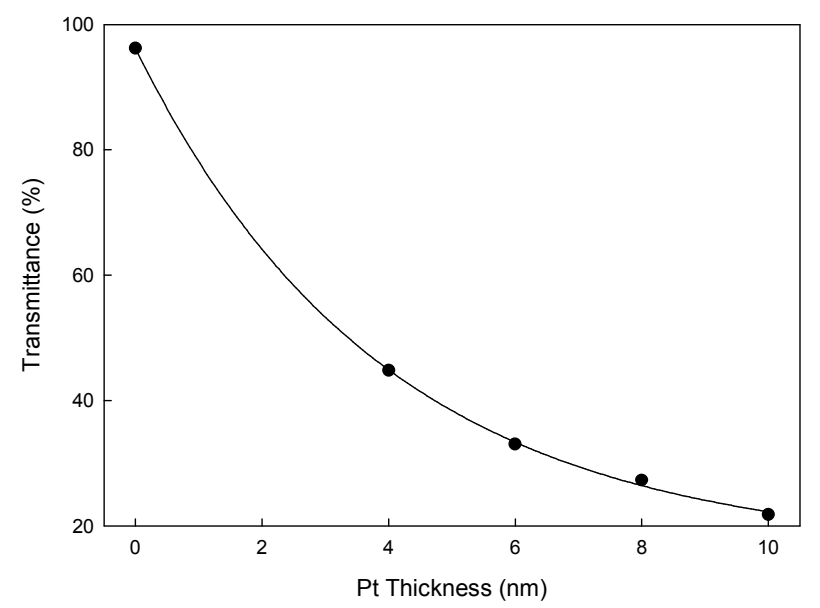

Fig. 4. Transmittance of GZO films without and with a surface Pt layer.

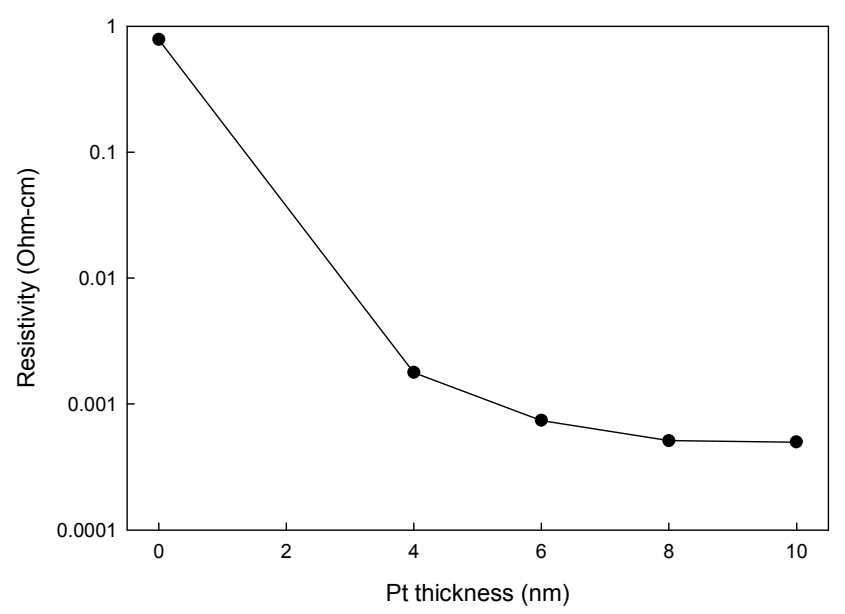

Fig. 3. Electrical resistivity of GZO films without and with a surface Pt layer

lower the optical transmittance is. This is attributed to the higher refractive index of the $\mathrm{Pt}$ film than that of the GZO thin film. The refractive indices of $\mathrm{Pt}$ films are shown in Fig. 5. When the difference in the refractive index is larger, the more the transmittance is reduced. The refractive index of the 22-nm GZO is 1.54 . Therefore, the refractive index difference between the $\mathrm{Pt}$ and GZO films reduces as the $\mathrm{Pt}$ thickness increases. This would have given increased transmittance with the Pt thickness but not decreased transmittance as shown in 
Fig. 4. The contradictory is lifted as the distinction coefficient of the Pt film is considered. As shown in Fig. 5, the distinction coefficient increases with the Pt thickness. A higher distinction coefficient leads to more absorption. Therefore the transmittance would then decrease with the Pt thickness from the view point of distinction coefficient. It is apparent that the effect of the distinction coefficient overrides that of the refractive index. As a result, the transmittance decreases with the Pt thickness, as

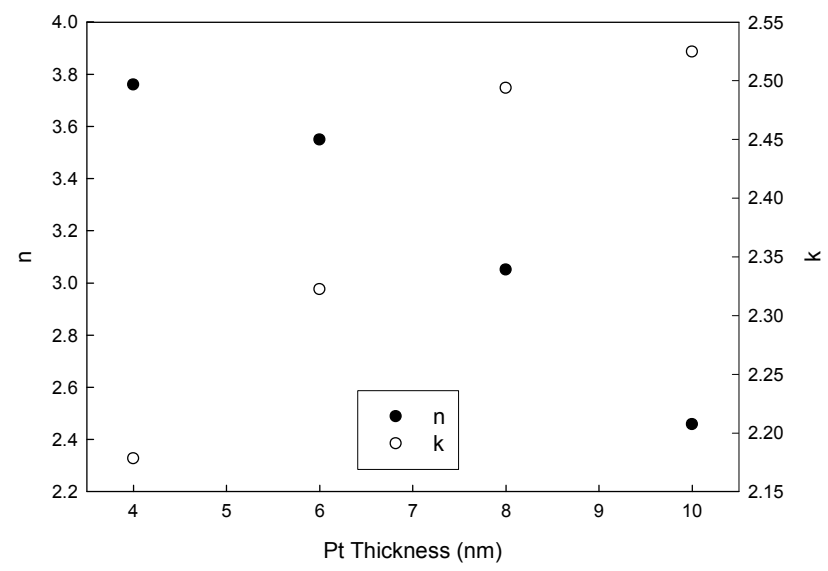

Fig. 5. The refractive index and distinction coefficient of Pt film at wavelength $=500 \mathrm{~nm}$.

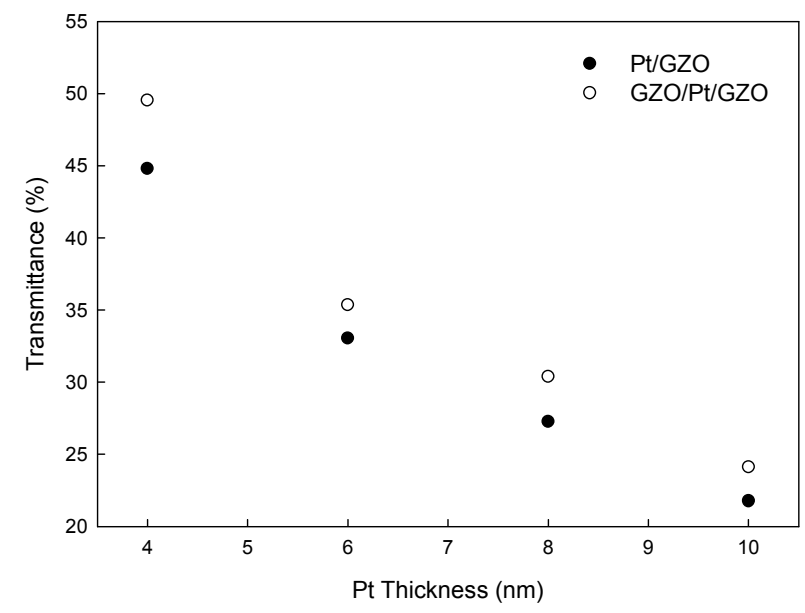

Fig. 6. Comparison of the optical transmittance of $\mathrm{Pt} / \mathrm{GZO}$ and $\mathrm{GZO} / \mathrm{Pt} / \mathrm{GZO}$ films. Each GZO has a thickness of $22 \mathrm{~nm}$ while the Pt thickness varies.

shown in Fig.4.

To improve the optical transmittance, an anti-refractive nanoscale GZO thin film is then deposited on Pt/GZO. As shown in Fig. 6, the optical transmittance is improved. However, the improvement is limited. The best optical transmittance, near 50\%, occurs for the 22-nm GZO/4-nm Pt/22-nm GZO thin film. Theoretical calculations similar to that mentioned above suggest the thickness of the GZO outer layer plays a critical role[24]. A GZO outer layer with a thickness between $58 \mathrm{~nm}$ to $68 \mathrm{~nm}$ and a 4-nm thick Pt mid-layer were determined to be optimal[25]. Therefore, a 66-nm GZO/4-nm Pt/66-nm GZO thin film was prepared and measured for its optical transmittance. The result is given in Fig. 7. For comparison, the optical transmittance of a $22-\mathrm{nm}$ $\mathrm{GZO} / 4-\mathrm{nm} \mathrm{Pt} / 22-\mathrm{nm}$ GZO tri-layer thin film is also given. An apparent improvement is seen. The optical transmittance in the visible range is greater than $75 \%$. This film also exhibits a low electrical resistivity in the order of $10^{-4} \mathrm{ohm}-\mathrm{cm}$.

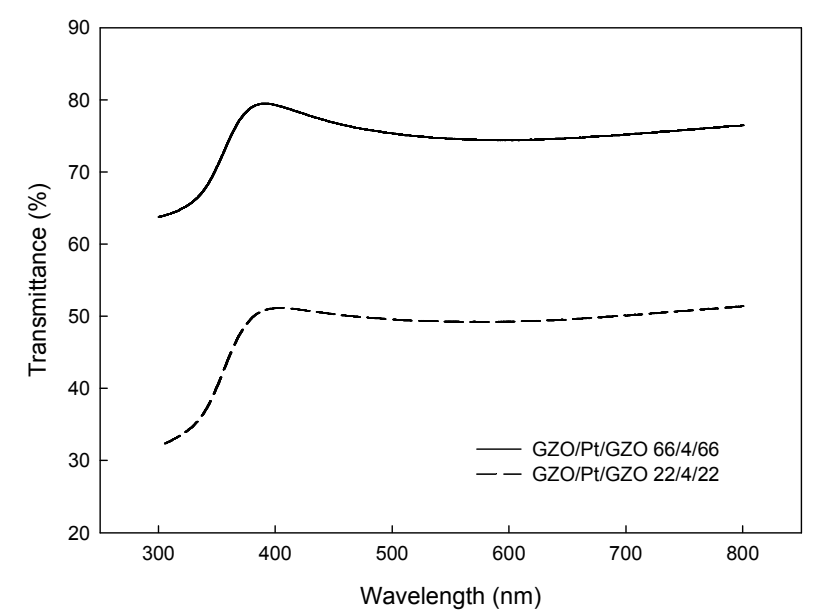

Fig. 7. Optical transmittances of $\mathrm{GZO} / \mathrm{Pt} / \mathrm{GZO}$ thin films. 


\section{Summary}

We have investigated nanoscale GZO thin films, bi-layer Pt/GZO thin films, and tri-layer $\mathrm{GZO} / \mathrm{Pt} / \mathrm{GZO}$ thin films for their characteristics. GZO thin films exhibit optical transmittances greater than $80 \%$ in the visible range. The optical transmittance depends on the film thickness. Although the optical transmittance is high, the electrical resistivity is also high, in the order of $10^{-1}$ to $10^{-2} \Omega-\mathrm{cm}$. A $\mathrm{Pt}$ surface layer is introduced to form bi-layer Pt/GZO films. The electrical resistivity is therefore largely reduced by 2 to 3 orders of magnitude. While the electrical resistivity is reduced, the optical transmittance is also reduced. The optical transmittance of Pt/GZO thin film is then enhanced by using an additional surface anti-refractive layer of GZO film. Through optical simulations, a GZO/Pt/GZO film exhibiting visible light transmittance greater than $75 \%$ and electrical resistivity in the order of $10^{-4}$ ohm-cm is obtained.

\section{Acknowledgement}

This work was supported by the National Science Council in Taiwan under Grant Nos. NSC 942622-E-006-036 and NSC 95-2120-M-006 -005.

\section{References}

[1] A. Suzuki, T. Matsushita, T. Aoki, A. Mori and M. Okuda: Thin Solid Films Vol.411 (2002), p. 23

[2] T. Minami: Semicond. Sci. Technol. Vol.20 (2005), p.S35

[3] Hai-Ning Cui, V. Teixeira and A. Monteiro: VacuumVol. 67 (2002), p.589

[4] B. Rech, O. Kluth, T. Repmann, T. Roschek, J. Springer, J. Müller, F. Finger, H. Stiebig and H. Wagner: Sol. Energy Mater. Sol. Cells Vol.74 (2002), p. 439

[5] V. Assuncão, E. Fortunato, A. Marques, A. Goncalves, I. Ferreira, H. Aguas and R. Martins: Thin Solid Films Vol.442 (2003), p.102

[6] H. J. Ko, Y. F. Chen, S. K. Hong, H. Wenisch, and T. Yao: Appl. Phys. Lett. Vol.77 (2000), p.3761

[7] Brian G. Lewis and David C. Paine: MRS Bulletin Vol.25 (2000), p. 22

[8] G. Leftheriotis, S. Papaefthimiou and P. Yianoulis: Solid State Ionics Vol.136 (2000), p.655

[9] Liu. Xuanjie, Cai Xun, Mao Jifang and Jin Chengyu: Applied Surface Science Vol.183 (2001), p.103

[10] Liu Xuanjie, Cai Xun, Qiao Jinshuo, Mao Jifang and Jiang Ning: Thin Solid Films Vol.441 (2003), p. 200

[11] K.H. Choi, J.Y. Kim, Y.S. Lee and H.J. Kim:Thin solid Films Vol.341 (1999), p.152

[12] A. Klöppel, W. Kriegseis, B.K. Meyer, A. Scharmann, C. Daube, J. Stollenwerk and J. Trube: Thin Solid Films Vol.365 (2000),1p.39

[13] Yuki Aoshima, Masami Miyazaki, Kazuo Sato, Yasuhiko Akao, Satoru Takaki and Kunihiko Adachi: Jpn. J. Appl. Phys. Vol.40 (2001), p.4166

[14] Andreas Klöppel, Bernd Meyer, Trube Jutta: Thin Solid Films Vol. 392 (2001), p.311 
[15] Jyh-Ming Ting and Chia-Kang Lin: J Am Ceram Soc. Vol. 89 (2006) p.3676

[16] Cheng-Chung Lee, Shang-Hui Chen, and Cheng-chung Jaing: Applied Optics, Vol.35 (1996), p.5698

[17] G. Leftheriotis, P. Yianoulis and D. Patrikios: Thin Solid Films Vol.306 (1997), p.92

[18] Seigo Ito, Tetsuya Takeuchi, Terumasa Katayama, Masato Sugiyama, Mizuho Matsuda, Takayuki Kitamura, Yuji Wada, and Shozo Yanagida: Chem. Mater. Vol.15 (2003), p.2824

[19] Yeon Sik Jung, Yong Won Choi, Ho Chul Lee, Dong Wook Lee: Thin Solid Films Vol.440 (2003), p. 278

[20] M. Bender, W. Seelig, C. Daube, H. Frankenberger, B. Ocker and J. Stollenwerk: Thin Solid Films Vol.326 (1998), p.67

[21] W. D. Westwood: J.Vac. Sci. Technol. Vol.15 (1978), p1

[22] D.E. Clark, F.D. Gac and W.H. Sutton: Microwaves: Theory and Application in Materials Processing, Ceramic Tranactions Vol. 21, the American Ceramic Society, Westville, OH, 1991

[23] E.J.J. Martin, M. Yan, M. Lane, J. Ireland, C.R. Kannewurf and R.P.H. Chang: Thin Solid Films Vol.461 (2004), p.309

[24] Ching-Hsuang Cheng: Characteristics and optical simulations of GZO/Pt/GZO transparent conductive multilayer thin film, MS thesis, National Cheng Kung University, Taiwan, 2006.

[25] Ching-Hsuang Cheng and Jyh-Ming Ting: Thin Solid Films Vol. 516 (2007), p.203 